\title{
Wavelet Collocation Method for Solving Elliptic Singularly Perturbed Problem
}

\author{
Bin Lin \\ School of Mathematics and Statistics, Lingnan Normal University, Zhanjiang, China \\ Email: linbin167@163.com
}

How to cite this paper: Lin, B. (2019) Wavelet Collocation Method for Solving Elliptic Singularly Perturbed Problem. Journal of Applied Mathematics and Physics, 7, 166-171.

https://doi.org/10.4236/jamp.2019.71014

Received: December 25, 2018

Accepted: January 19, 2019

Published: January 22, 2019

Copyright ( 2019 by author(s) and Scientific Research Publishing Inc. This work is licensed under the Creative Commons Attribution International License (CC BY 4.0).

http://creativecommons.org/licenses/by/4.0/

\section{(c) (†) Open Access}

\begin{abstract}
Wavelet collocation method is used to solve an elliptic singularly perturbed problem with two parameters. The B-spline function is used as a single mother wavelet, which leads to a tri-diagonal linear system. The accuracy of the proposed method is demonstrated by test problem and the result shows the reliability and efficiency of the method.
\end{abstract}

\section{Keywords}

B-Spline Functions, Wavelet Collocation Method, Elliptic Singularly

Perturbed Problems

\section{Introduction}

Wavelet functions are finite-energy functions with well localization properties. Any function of $L^{2}(R)$ can be expressed by the dilation and translation of wavelet functions. Wavelet analysis has been found success in many science and engineering. The stiffness matrix is sparse when wavelet functions is used as trial functions, so wavelet methods are especially suitable for singular perturbed and a local severe gradients problem. Wavelets have many excellent properties such as orthogonality, compact support, exact representation, flexibility to represent functions at different levels of resolution.

In the paper, we consider the following an elliptic singularly perturbed problem with two parameters.

$$
\begin{gathered}
L u:=-\varepsilon_{1} \Delta u+\varepsilon_{2} a(x) u_{x}+b(x) u_{y}+c(x) u=f(x, y), \text { in } \Omega \\
u=0, \text { on } \partial \Omega
\end{gathered}
$$

where $\Omega=(0,1) \times(0,1), \varepsilon_{1}, \varepsilon_{2}$ is a small positive parameter, $a(x), b(x)$ and $c(x)$ are sufficiently smooth real-valued functions. 
This problem, arising in chemical flow reactor theory [1] as well as in case of boundary layers controlled by suction (or blowing) of some fluid [2], has been studied by several authors [3] [4] [5] [6]. The former research is already done a lot of work, but we want to improve it. It is so attractive to mathematicians due to the fact that modeling of physical problems often requires the solution of boundary value problems with many small parameters. It is well known that usual numerical treatment of singular perturbation problems gives major computational difficulties and fail to give accurate solutions. The former research is already done a lot of work, but we want to improve it further improved

The aim of this paper is to given a wavelet collocation method to solve an elliptic singularly perturbed problem with two parameters, the $3^{\text {rd }}$ order B-spline function is used as a single mother wavelet to construct the numerical method. This method is tested for its efficiency by handling an example.

\section{Construction of Wavelet Collocation Method}

\subsection{Spline Scaling Function}

The expression formula of the $3^{\text {rd }}$ order B-spline function $N_{4}(x)$ is

$$
N_{4}(x)=\int_{0}^{1} N_{3}(x-\tau) \mathrm{d} \tau= \begin{cases}\frac{1}{6} x^{3} & {[0,1)} \\ -\frac{1}{2} x^{3}+2 x^{2}-2 x+\frac{2}{3} & {[1,2)} \\ \frac{1}{2} x^{3}-4 x^{2}+10 x-\frac{22}{3} & {[2,3)} \\ -\frac{1}{6} x^{3}+2 x^{2}-8 x+\frac{32}{3} & {[3,4)} \\ 0 & \text { others }\end{cases}
$$

The $3^{\text {rd }}$ order B-spline function $N_{4}(x)$ is usually used to calculate in practice, and is easy and efficient.

\subsection{Tensor Product}

Supposing that one-dimensional scaling functions $\phi^{1}(x), \phi^{2}(x)$ generate multi-resolution analyses $\left\{V_{j}^{1}\right\},\left\{V_{j}^{2}\right\}$ respectively, the tensor product space of $V_{j}^{1}$ and $V_{j}^{2}$ is

$$
V_{j}=V_{j}^{1} \otimes V_{j}^{2}
$$

where $\otimes$ is the Kroneck symbol, the span of $V_{j}^{1}$ is $\left\{2^{j / 2} \phi^{1}\left(2^{j}-k\right)\right\}$ and that of $V_{j}^{2}$ is $\left\{2^{j / 2} \phi^{2}\left(2^{j}-l\right)\right\}$.

Two-dimensional functions are written in the form

$$
\begin{gathered}
f_{j, k, l}(x, y)=2^{j / 2} f\left(2^{j} x-k, 2^{j} y-l\right) \\
\phi(x, y)=\phi^{1}(x) \phi^{2}(y)
\end{gathered}
$$

The $\left\{\phi_{j, k, l}(x, y) ; k, l \in Z\right\}$ is the span of $V_{j}$ and $\left\{V_{j}\right\}$ generates a multiresolution analysis of $L^{2}\left(R^{2}\right)$ and $\phi(x, y)$ are scaling functions correspon- 
dingly. The $3^{\text {rd }}$ order B-spline function $N_{4}(x)$ was selected as the basis, then the two dimensional scaling function is

$$
\phi(x, y)=\phi^{1}(x) \phi^{2}(y)=N_{4}(x) N_{4}(y)
$$

\subsection{Construct of the Basis Function}

We consider two dimensional functions $f(x, y)$ and select the two dimensional scaling functions as the basis functions. A square domain $[0,1] \times[0,1]$ is separated by a uniform grid with intervals $N=2^{j}$, the grid sizes are denoted by $h=1 / 2^{j},(j \in Z)$, and the grid points are denoted by

$$
\begin{aligned}
& x_{m}=m h, m=0,1,2, \cdots, 2^{j} \\
& y_{n}=n h, n=0,1,2, \cdots, 2^{j}
\end{aligned}
$$

The approximated numerical values of $u$ at the grid point $\left(x_{m}, y_{n}\right)$ are denoted by $u_{m, n} \quad(m, n \in Z)$

The basis function is defined as

$$
\phi(x, y)=\phi^{1}(x) \phi^{2}(y)=N_{4}\left(\frac{x-x_{i}}{h}\right) N_{4}\left(\frac{y-y_{i}}{h}\right)
$$

\subsection{Solve an Elliptic Singularly Perturbed Problem}

In the proposed algorithm, the $3^{\text {rd }}$ order B-spline function $N_{4}(x, y)$ is used as a single mother wavelet, i.e. $\phi(x, y)=N_{4}(x, y)$ and dilation and translation of mother wavelet functions can construct any function of $L^{2}\left(R^{2}\right)$.

$$
S(x, y)=\sum_{j_{1}=-1}^{N+1} \sum_{j_{2}=-1}^{N+1} C_{j_{1}, j_{2}} N_{4}\left(\frac{x-x_{j_{1}}}{h}\right) N_{4}\left(\frac{y-y_{j_{2}}}{h}\right)
$$

The approximated numerical values of $u$ at the grid point $\left(x_{m}, y_{n}\right)$ are denoted by

$$
S\left(x_{m}, y_{n}\right)=\sum_{j_{1}=-1}^{N+1} \sum_{j_{2}=-1}^{N+1} C_{j_{1}, j_{2}} N_{4}\left(\frac{x_{m}-x_{j_{1}}}{h}\right) N_{4}\left(\frac{y_{n}-y_{j_{2}}}{h}\right)
$$

Substituting (11) into Equation (1) for all the nodes, we can obtain the following linear equations

$$
\begin{aligned}
& \frac{-\varepsilon_{1}}{h^{2}} \sum_{j_{1}=-1}^{N+1} \sum_{j_{2}=-1}^{N+1} C_{j_{1}, j_{2}}\left[N_{4}^{\prime \prime}\left(\frac{x_{m}-x_{j_{1}}}{h}\right) N_{4}\left(\frac{y_{n}-y_{j_{2}}}{h}\right)+N_{4}\left(\frac{x_{m}-x_{j_{1}}}{h}\right) N_{4}^{\prime \prime}\left(\frac{y_{n}-y_{j_{2}}}{h}\right)\right] \\
& +\frac{1}{h} \sum_{j_{1}=-1}^{N+1} \sum_{j_{2}=-1}^{N+1} C_{j_{1}, j_{2}}\left[\varepsilon_{2} a(m h) N_{4}^{\prime}\left(\frac{x_{m}-x_{j_{1}}}{h}\right) N_{4}\left(\frac{y_{n}-y_{j_{2}}}{h}\right)\right. \\
& \left.+b(m h) N_{4}\left(\frac{x_{m}-x_{j_{1}}}{h}\right) N_{4}^{\prime}\left(\frac{y_{n}-y_{j_{2}}}{h}\right)\right] \\
& +c(m h) \sum_{j_{1}=-1}^{N+1} \sum_{j_{2}=-1}^{N+1} C_{j_{1}, j_{2}} N_{4}\left(\frac{x_{m}-x_{j_{1}}}{h}\right) N_{4}\left(\frac{y_{n}-y_{j_{2}}}{h}\right)=f\left(x_{m}, y_{n}\right) \quad \text { in } \Omega \\
& \sum_{j_{1}=-1}^{N+1} \sum_{j_{2}=-1}^{N+1} C_{j_{1}, j_{2}} N_{4}\left(\frac{x_{m}-x_{j_{1}}}{h}\right) N_{4}\left(\frac{y_{n}-y_{j_{2}}}{h}\right)=0 \text { on } \partial \Omega
\end{aligned}
$$


Above linear equations are written in matrix form as

$$
B \cdot C=F
$$

The B-spline function is a useful wavelet basis function, the stiffness matrix is sparse. When it is used as trial functions, which leads to a tri-diagonal linear system. Hence the wavelet collocation method using the $3^{\text {rd }}$ order B-spline function $N_{4}(x, y)$ as a basis function applied to an elliptic singularly perturbed problem has a unique solution $S(x, y)$ given by Equation (10).

\section{Numerical Results}

In the section, we illustrate the numerical techniques discussed in the previous section by the following problems.

The point-wise errors are given by

$$
E\left(x_{i}\right)=\left|S\left(x_{i}\right)-u\left(x_{i}\right)\right|
$$

For every $\varepsilon$ the computed maximum point-wise errors are given by

$$
E^{N}=\max _{0 \leq i \leq N}\left|u\left(x_{i}\right)-S\left(x_{i}\right)\right|
$$

A test problem [6] is given by

$$
L u:=-\varepsilon_{1} \Delta u+\varepsilon_{2}(3-x) u_{x}+u=f(x, y), \text { in } \Omega=(0,1) \times(0,1)
$$

with the boundary conditions

$$
u=0 \text { on } \partial \Omega
$$

The analytical solution is given by

$$
u(x, y)=\frac{1}{4}\left(1-\mathrm{e}^{\frac{-\varepsilon_{2} k_{1} x}{2 \varepsilon_{1}}}\right)\left(1-\mathrm{e}^{\frac{-\varepsilon_{2} k_{2}(1-x)}{2 \varepsilon_{1}}}\right)\left(1-\mathrm{e}^{\frac{-y}{\sqrt{\varepsilon_{1}}}}\right)\left(1-\mathrm{e}^{\frac{y-1}{\sqrt{\varepsilon_{1}}}}\right),
$$

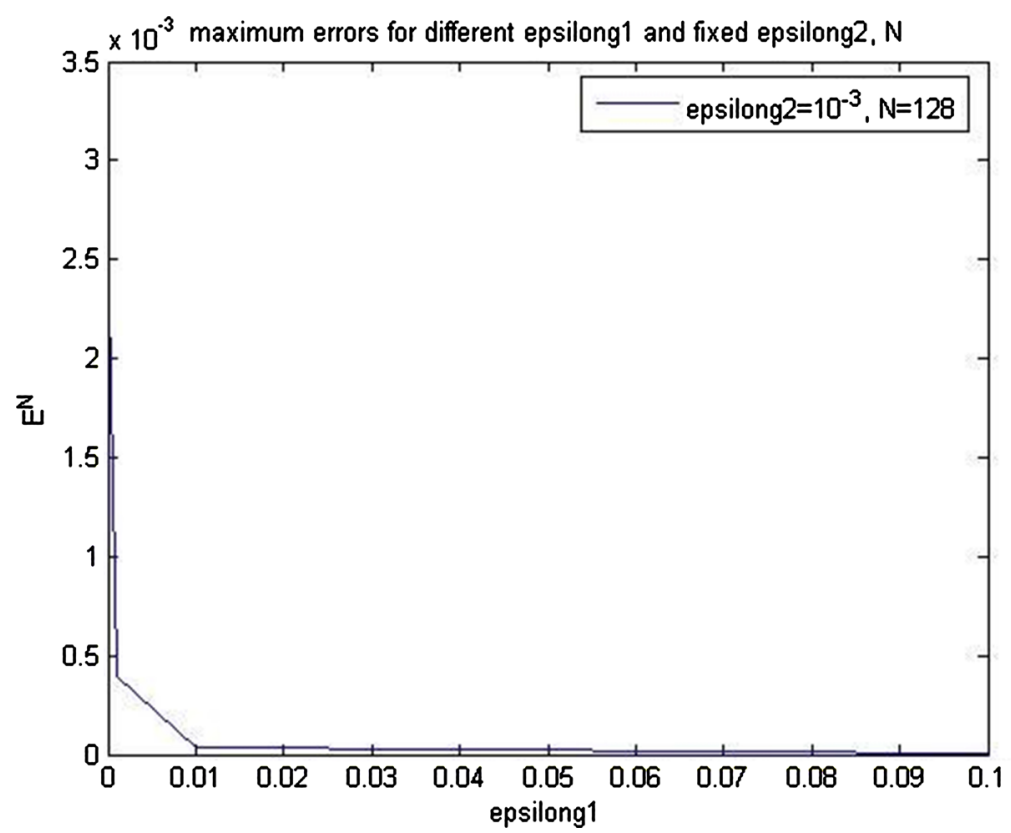

Figure 1. Relation of maximum point-wise errors and values of $\mathcal{\varepsilon}$. 


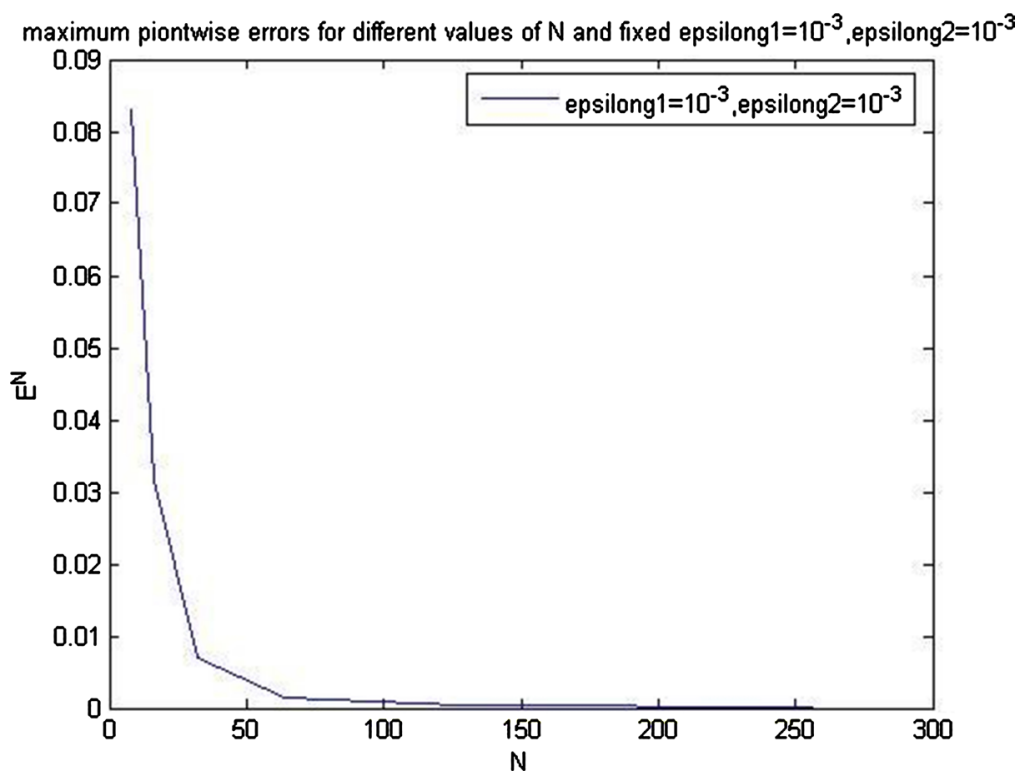

Figure 2. Relation of maximum point-wise errors and values of $N$.

where $k_{1}=-1+\sqrt{1+16 \varepsilon_{1} / \varepsilon_{2}^{2}}, k_{2}=1+\sqrt{1+16 \varepsilon_{1} / \varepsilon_{2}^{2}}$,

Relation of maximum point-wise errors and values of $\varepsilon$ and $N$ is given in Figure 1 and Figure 2.

It observed that

1) when $\varepsilon_{1} \in(0,0.01]$ increases for fixed $\varepsilon_{2}$ and $N$, the maximum point-wise errors decrease rapidly;

when $\varepsilon_{1} \in(0.01,1)$ increases for fixed $\varepsilon_{2}$ and $N$, the maximum point-wise errors decreases slowly to 0 ;

2) when $N<50$ increase for fixed $\varepsilon_{1}$ and $\varepsilon_{2}$, the maximum point-wise errors decreases rapidly;

when $N>50$ increase for fixed $\varepsilon_{1}$ and $\varepsilon_{2}$, the maximum point-wise errors decreases slowly to 0 ;

\section{Conclusion}

The numerical results show clearly the effect of $\varepsilon$ and $N$ on the solution of an elliptic singularly perturbed problem and the present method is relatively simple and it is applicable technique and approximates the exact solution very well.

\section{Acknowledgements}

The authors would like to thank the editor and the reviewers for their valuable comments and suggestions to improve the results of this paper.

This work was supported by the Natural Science Foundation of Guangdong (No. 2015A030313827) and the Key Subject Program of Lingnan Normal University [No. 1171518004].

\section{Conflicts of Interest}

The author declares no conflicts of interest regarding the publication of this pa- 
per.

\section{References}

[1] Jain, P.C., Shankar, R. and Bhardwaj, D. (1997) Numerical Solution of the Korteweg-Devries (KdV) Equation. Chaos, Solitons \& Fractals, 8, 943-951.

[2] Schlichting, H. (1979) Boundary Layer Theory. 7th Edition, McGraw-Hill, New York.

[3] López, J.L., Sinusía, E.P. and Temme, N.M. (2006) First Order Approximation of an Elliptic 3D Singular Perturbation Problem. Studies in Applied Mathematics, 116, 303-319.

[4] Shih, S.D. (1996) A Novel Uniform Expansion for a Singularly Perturbed Parabolic Problem with Corner Singularity. Methods and Applications of Analysis, 3, $203-$ 227. https://doi.org/10.4310/MAA.1996.v3.n2.a3

[5] O’Riordan, E., Pickett, M.L. and Shishkin, G.I. (2006) Numerical Methods for Singularly Perturbed Elliptic Problems Containing Two Perturbation Parameters. $\mathrm{Ma}$ thematical Modelling and Analysis, 11, 199-212.

[6] Teofanov, L.J. and Roos, H.-G. (2008) An Elliptic Singularly Perturbed Problem with Two Parameters II: Robust Finite Element Solution. Journal of Computational and Applied Mathematics, 212, 374-389. https://doi.org/10.1016/j.cam.2006.12.013 